\title{
LUMINESCENCE DU BENZĖNE EXCITÉ DANS DES MATRICES D'AZOTE ET D'ARGON SOLIDES
}

\author{
E. Faure, F. Valadier, R. Bergeon et J. Janin \\ Laboratoire de Spectroscopie et de Luminescence, Université de Lyon
}

\begin{abstract}
Résumé. - Le benzène excité par l'azote actif au voisinage de 4 et $20^{\circ} \mathrm{K}$ émet avec une forte intensité le système $T_{1} \rightarrow S_{0}$, chacune des bandes observées étant constituée de trois composantes dont les intensités relatives dépendent de la température. Les essais ont porté sur du benzène en solution dans des matrices d'azote et d'argon de différentes compositions et on a comparé pour chaque mélange le spectre obtenu par ce mode d'excitation avec celui du benzène irradié par l'ultraviolet. On en a conclu que la composante de plus courte longueur d'onde peut être attribuée à des molécules fixées dans l'azote $\beta$ tandis que la composante centrale et probablement aussi celle de plus grande longueur d'onde sont dues au benzène dilué dans l'azote $\alpha$.
\end{abstract}

Abstract. - Benzene molecules, when excited by active nitrogen in the neighbourhood of 4 and $20^{\circ} \mathrm{K}$, emit the $T_{1} \rightarrow S_{0}$ system with strong intensity; each band so observed exhibits three components, with relative intensities depending on temperature. The experiments were made on benzene trapped in nitrogen and argon matrices with different concentrations, and for each mixture the spectrum obtained by this typc of excitation was compared with that of benzene irradiated by UV light. It was concluded that the shortest wave length component can be attributed to molecules trapped in $\beta$-nitrogen, whereas the central component, and probably the longest wave length one too, may be due to benzene trapped in $x$-nitrogen.

On a signalé dans une note récente [1] que le benzène excité par l'azote actif au voisinage de 4 et de $20^{\circ} \mathrm{K}$ émet avec une forte intensité le système $T_{1} \rightarrow S_{0}$. Les bandes observées sont constituées de trois composantes $A, B$ et $C$ dont les intensités relatives dépendent de la température et de la concentration du benzène. Pour expliquer l'origine de ces composantes on a admis l'existence dans la matrice solide des deux phases $\alpha$ et $\beta$ de l'azote. Dans le but de contrôler la validité de cette hypothèse on a procédé aux essais suivants :

1) Des mélanges d'azote et d'argon de concentrations variables étaient excités dans les conditions indiquées précédemment [1], puis après l'introduction d'une faible quantité de benzène ( $1 \%$ en volume), ils étaient condensés à la température de l'hélium ou de l'hydrogène liquide.

Les spectres obtenus diffèrent peu de ceux fournis par les mélanges binaires azote-benzène; on constate toutefois une modification des intensités relatives des composantes lorsque les proportions d'azote et d'argon varient. C'est ainsi que pour des teneurs en argon comprises entre 10 et $50 \%$ un renversement d'intensité des composantes $A$ et $B$ se manifeste sur les spectrogrammes enregistrés vers $20^{\circ} \mathrm{K}$ (Fig. 1) et pendant l'exaltation violette qui se produit au cours du réchauffement du dépôt solide à partir de $4{ }^{\circ} \mathrm{K}$ (Fig. 2) ; la composante $A$ est alors prédominante.
Lorsque la teneur en argon augmente, les bandes s'élargissent et deviennent plus diffuses et pour les fortes concentrations il apparait de nouvelles composantes. Ainsi, quand le mélange contient $80 \%$ d'argon, l'arête $A$ se dédouble en deux composantes $A_{1}$ et $A_{2}$ d'égale intensité (Fig. 3). Alors que $A_{2}$ occupe sensiblement la même position que l'arête primitive $A$, la composante $A_{1}$ est située à $25 \mathrm{~cm}^{-1}$ de cette dernière du côté des courtes longueurs d'onde. Pour les concentrations voisines de $95 \%$, on distingue une troisième composante de faible intensité $A_{3}$ (Fig. 4). C'est cette dernière que l'on observe dans les spectres de phosphorescence du benzène dilué dans des matrices d'argon solide [2].

On constate, en outre, que pour de tels mélanges l'arête $B$ est dédoublée ; les deux composantes $B_{1}$ et $B_{2}$ semblent correspondre respectivement à celles qui apparaissent dans les spectres du benzène piégé dans l'azote et dans l'argon purs [2], mais elles sont déplacées par rapport à ces dernières de 10 à $15 \mathrm{~cm}^{-1} \mathrm{du}$ côté des courtes longueurs d'onde.

On doit noter enfin que la présence d'argon a également pour effet de faire disparaître la composante $C$ que l'on observe dans l'émission des mélanges binaires azote-benzène de même concentration en benzène.

2) On a soumis un mélange d'azote et de benzène condensé à 4 ou à $20^{\circ} \mathrm{K}$ au rayonnement d'une lampe 


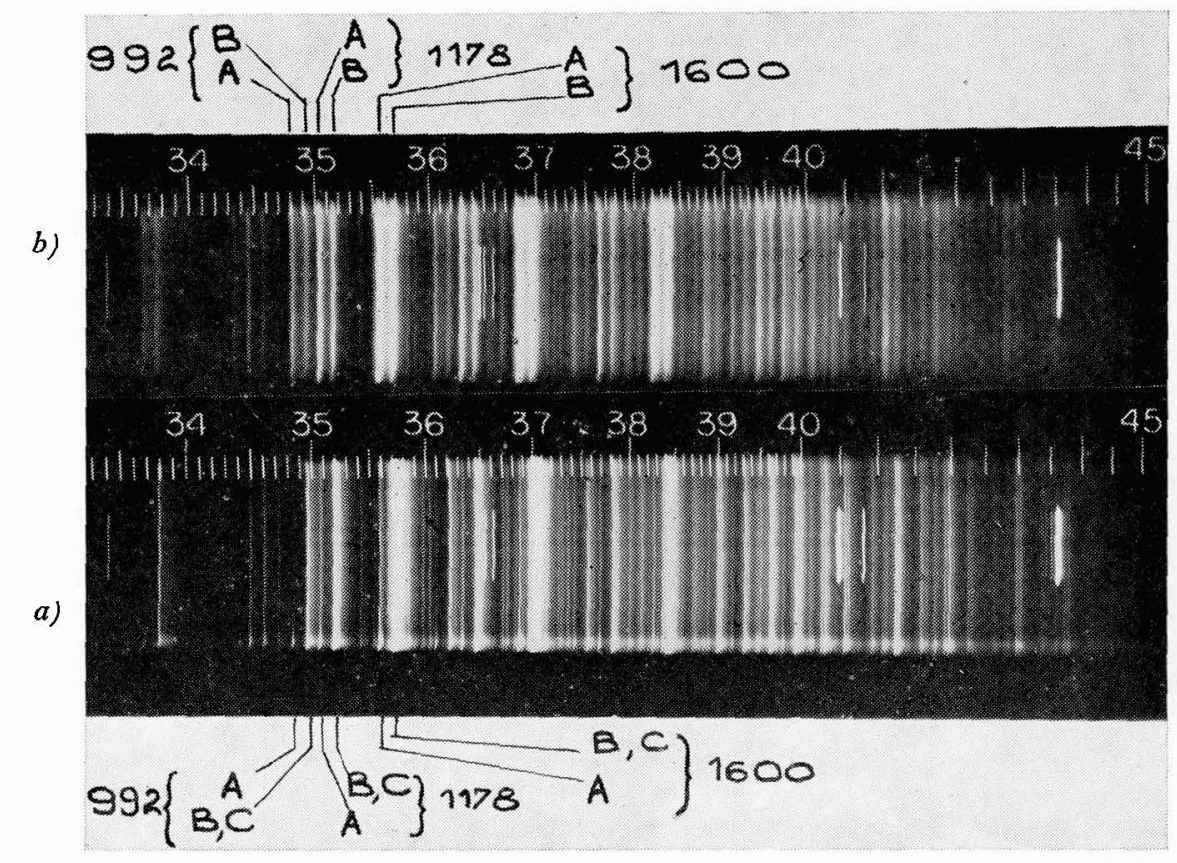

Fig. 1. - a) Emission du benzène dans une matrice d'azote à 4 \% $\mathrm{K}$.

b) Emission du benzène dans une matrice d'azote et d'argon $(25 \%)$ à $20 \% \mathrm{~K}$.
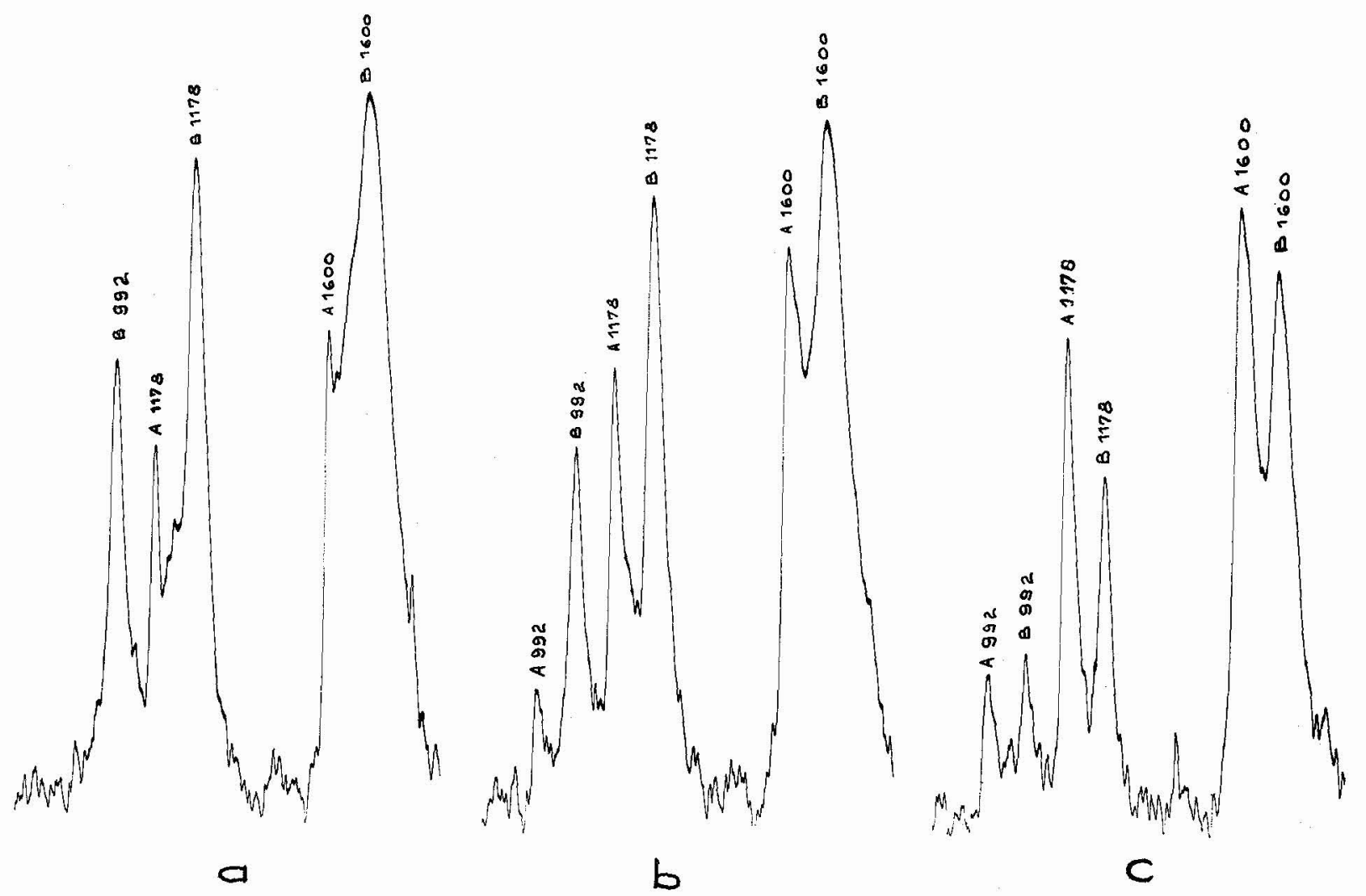

FIG. 2. - Evolution de l'intensité des différentes composantes des bandes $v=992,1178$ et $1600 \mathrm{~cm}^{-1}$ pendant la période de réchauffement, pour un mélange de composition $74 \% \mathrm{~N}_{2}, 25 \% \mathrm{~A}, 1 \% \mathrm{C}_{6} \mathrm{H}_{6}$. a) Première partie du réchauffement. b) Début de la période d'exaltation de l'émission violette. c) Fin de cette période. 


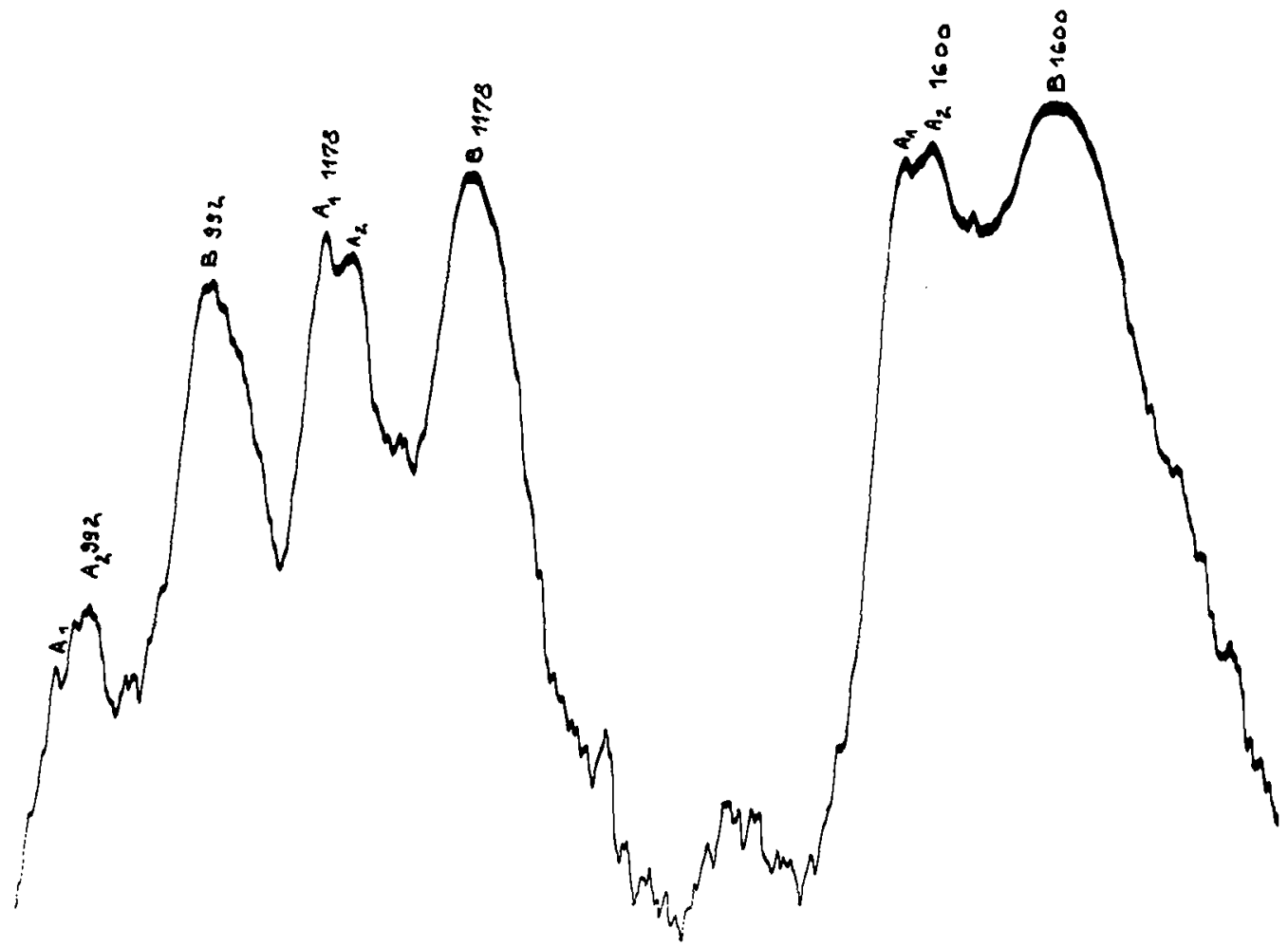

Fig. 3. - Emission à $20 \% \mathrm{~K}$ d'un mélange $\mathrm{N}_{2}(20 \%)$, A $(79 \%), \mathrm{C}_{6} \mathrm{H}_{6}(1 \%)$.

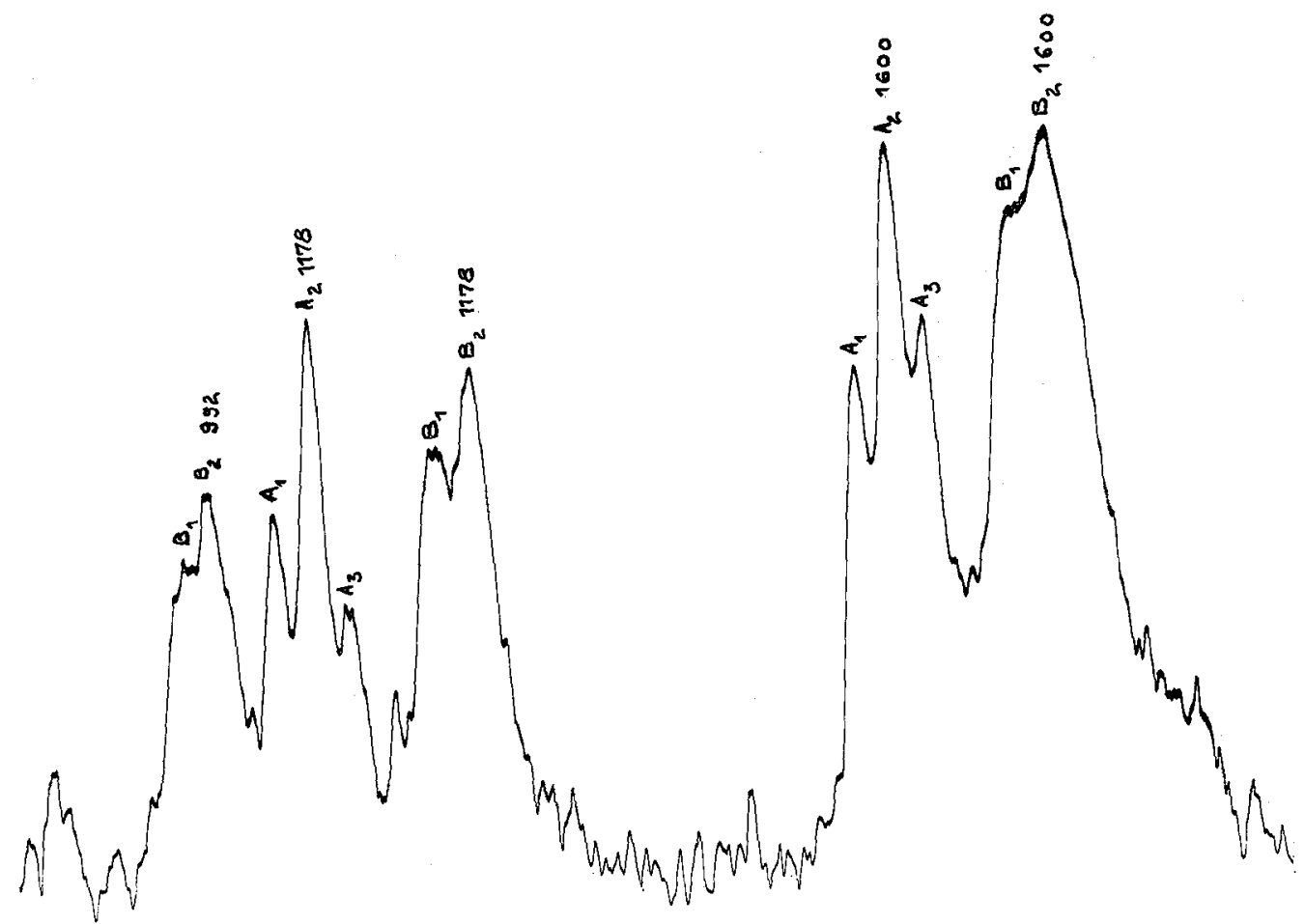

FIG. 4. - Emission à $20 \% \mathrm{~K}$ d'un mélange $\mathrm{N}_{2}(5 \%), \mathrm{A}(94 \%), \mathrm{C}_{6} \mathrm{H}_{6}(1 \%)$. 
à vapeur de mercure sous basse pression. La luminescence du dépôt solide est analysée comme dans les expériences précédentes au moyen d'un spectrographe médium Hilger à optique de quartz. En utilisant une fente relativement large $(0,04 \mathrm{~mm})$ et en posant assez longtemps ( 3 heures environ), on a obtenu des spectrogrammes de densité comparable à celle des enregistrements effectués avec le premier procédé d'excitation.

On constate qu'à $4{ }^{\circ} \mathrm{K}$ le spectre de phosphorescence du benzène comporte uniquement les deux composantes $B$ et $C$, cette dernière nettement plus faible que $B$ n'étant d'ailleurs visible que dans le cas des progressions de forte intensité. L'aspect du système demeure inchangé lorsqu'on fait varier la concentration du benzène entre 0,1 et $5 \%$. A $20{ }^{\circ} \mathrm{K}$, les spectres obtenus sont analogues; toutefois la composante $A$ apparait faiblement dans les progressions les plus intenses pour les mélanges de teneur en benzène supérieure à $1 \%$.

Lorsque la matrice est constituée d'un mélange d'azote et d'argon, on n'observe plus la composante $C$ tandis qu'à $20^{\circ} \mathrm{K}$ et pour des teneurs en argon de l'ordre de $30 \%$, l'intensité de la composante $A$ est supérieure à celle qu'elle avait dans les expériences similaires réalisées avec les mélanges azote-benzène ; néanmoins elle n'atteint pas celle de la composante $\mathrm{B}$, contrairement à ce qui se produit lorsqu'on excite le même mélange par l'azote actif. Cette différence est probablement due au fait que dans ce cas, la température du dépôt solide est plus élevée. Enfin, lorsque la matrice contient de fortes proportions d'argon $(80 \%)$ l'arête $A$ disparait.

Ces deux séries d'observations qui montrent l'influence de la température et celle de l'argon sur l'intensité de la composante $A$, renforcent l'hypothèse précédemment avancée, selon laquelle l'émission de cette radiation doit être attribuée à des molécules de benzène fixées dans la phase hexagonale $\beta$ de l'azote. On sait, en effet, que cette dernière est favorisée par la présence d'argon qui abaisse le point de transformation $\beta-\alpha$ (3). Ce seraient au contraire des molécules piégées dans la phase $\alpha$ qui seraient responsables de l'émission des composantes $B$ et $C$. Quant aux arêtes qui apparaissent lorsque le mélange contient des proportions élevées d'argon, elles sont probablement dues aux molécules de benzène condensées dans une matrice d'argon solide (ce qui serait le cas de $A_{3}$ et de $B_{2}$ ) ou dans des phases mixtes argon-azote.

\section{Bibliographie}

[1] Faure (E.), Valadier (F.), Bergeon (R.) et Janin (J.) C. R. Acad. Sc., 1966, 263, p. 1234.

[2] Diamant (Y.), Hexter (R. M.) and Schnepp (O.), $J$. Mol. Spectr., 1965, 18, p. 158.

[3] Barrett (C. S.) and Meyer (L.), J. chem. phys., 1964, 42, p. 107.

\section{DISCUSSION}

R. Lopez-Delgado.. - A propos de l'activité de la vibration $v_{1}\left(a_{1 g}\right)$ en phosphorescence, j'ai à faire la remarque suivante : la bande que l'on observe à environ $0,0-990 \mathrm{~cm}^{-1}$ n'est pas due exclusivement à l'activité de la vibration $a_{1 \mathrm{~g}}\left(992 \mathrm{~cm}^{-1}\right)$, car il y a superposition d'une autre vibration de symétrie $b_{2 \mathrm{~g}}$ et de fréquence $985 \mathrm{~cm}^{-1}$. En effet, notre étude de la phosphorescence du $C_{6} D_{6}$ (dans le cyclohexane) où cette quasi-coïncidence de fréquences n'est plus possible $\left(a_{1 \mathrm{~g}}, 943 \mathrm{~cm}^{-1} ; b_{2 \mathrm{~g}}, 827 \mathrm{~cm}^{-1}\right)$ montre que l'activité réelle de la vibration $v_{1}\left(a_{1 \mathrm{~g}}\right)$ est très faible (S. Leach et R. López-Delgado, J. Chim. Physique, 1964, 61, 1636).

D'autre part, la structure des spectres que vous observez indiquerait que le benzène se trouve dans des « sites interstitiels équivalents » (comme $M$. Leach les a décritsdans sa conférence), car il est très peu probable que le benzène occupe des "sites de substitution » dans la maille cristalline de l'azote. 